\title{
New Directions in Brain Imaging Research in Functional Gastrointestinal Disorders
}

\author{
Yehuda Ringel \\ UNC School of Medicine, Department of Medicine, Division of Digestive and Liver Diseases, Chapel Hill, N.C., USA
}

\begin{abstract}
Key Words
Brain imaging research, new directions · Functional gastrointestinal disorders • Functional brain imaging • FGID, brain imaging research
\end{abstract}

\begin{abstract}
Functional brain imaging has greatly enhanced the ability to investigate brain-gut interactions and to assess the central nervous system role on visceral pain perception. The results of studies using brain imaging in irritable bowel syndrome (IBS) have demonstrated differences in brain activation between patients with IBS and healthy controls. In addition, the more recent studies are starting to shed light on pathophysiological mechanisms that underlie the generation of functional gastrointestinal (GI) symptoms as well as the response to treatment. These studies highlight the potential of functional brain imaging to become an important and exciting investigative tool in research of functional GI disorders. However, the multifactorial, multideterminant nature of these disorders, the current limitations in the understanding of the pathophysiology of the disorders, and the heterogeneous patient population make brain imaging research in this field difficult and require caution in the interpretation of the data. The continued development of brain imaging techniques provides not only exciting opportu-
\end{abstract}

nities but also significant challenges to the field. This article focuses on brain imaging research in functional Gl disorders. It describes some of the recent developments in the use of brain imaging in research of the brain-gut axis and provides an overview of the current data.

Copyright $(2006$ S. Karger AG, Basel

\section{Introduction}

The pathophysiological mechanisms that underlie the various gastrointestinal (GI) symptoms associated with functional GI disorders (FGID) are still not clear. The current operational framework for understanding these symptoms is based on the combination of several contributing domains including GI functions such as motility and sensation, perception, and psychosocial factors, otherwise known as the biopsychosocial model [1]. According to this model, GI symptoms can result not only from a diseased or malfunctioning gut, but also from dysregulation at any level of the gut control mechanisms. This includes the enteric nerve systems, the autonomic and spinal neuronal pathways, and the brainstem and cerebral cortex (i.e. the brain-gut axis) [1, 2]. With regard to the involvement of the central nervous system (CNS) part of the brain-gut axis, there is an increasing body of evi-

\section{KARGER}

Fax +4161306 1234 E-Mail karger@karger.ch www.karger.com 
dence pointing to the importance of the CNS influence on the clinical presentation of FGID. Clinical and epidemiological studies have shown a high prevalence of psychosocial stressors and psychiatric co-morbidity in patients with FGID and have provided evidence for their effects on the onset of the disorders, their symptoms severity, and outcome [3, 4]. Physiologic studies are providing new insight into the pathophysiological mechanisms by which the CNS processes and modulates afferent information from the GI tract, how this influences GI motor and sensory functions, and the affective and behavioral responses to various intestinal stimulation [5]. However, physiologic and mechanistic research on the role of CNS in FGID is difficult since direct assessment of braingut axis function in humans is not feasible. Therefore researchers currently focus on brain stimulation and brain imaging techniques in order to better understand the cortical function in relation to intestinal function and FGID [6]. This article focuses on brain imaging research in FGID. It describes some of the recent developments in the use of brain imaging in research of the brain-gut axis and provides an overview of the current data.

\section{Functional Brain Imaging}

Recent developments in functional brain imaging techniques and their availability as investigative tools in clinical research have advanced research in FGID, most particularly with regard to the role of CNS and brain-gut axis in their pathophysiology. Functional brain imaging using positron emission tomography (PET), functional magnetic resonance imaging (fMRI), and magnetoencephalography (MEG) enables identification and localization of in vivo activity in the human brain with great anatomical precision.

By tracing the concentration and distribution changes of intravenously injected radiolabeled isotope in brain tissue, PET is able to measure changes in regional brain functions such as neuronal metabolism (18-fluorodeoxyglucose) or cerebral blood flow $\left(\mathrm{H}_{2}{ }^{15} \mathrm{O}\right)$. These changes are well correlated with regional neuronal brain activity [7]. Therefore, a computerized reconstruction can be used to create topographic images that express the relative changes in brain activation in response to various stimuli.

As with PET, fMRI is also able to measure changes in brain activity. The basic principle relies on the fact that during spurts of neuronal activity, the brain resorts to

New Directions in Brain Imaging

Research in FGID anaerobic metabolism. Thus, during neuronal activity there is an increase in regional cerebral blood flow but only a slight increase in the oxygen consumption. This causes an increase in the concentration of oxygenated hemoglobin in the area of activation. The change in the quantities of oxygenated and deoxygenated hemoglobin causes a change in the water proton magnetic field and therefore a change in the magnetic resonance signal [7]. Unlike early studies that have used a $1.5 \mathrm{~T}$, recent advances in high field technology and the increased availability of high field ( $3 \mathrm{~T}$ or more) human scanners have improved imaging abilities and opened the door for new applications of MRI in clinical research [8].

Although developments in PET and fMRI have been helpful in accurately identifying brain regions involved in processing intestinal sensory signals and pain, they do not have sufficient temporal resolution to enable mapping of cortical dynamic (millisecond-by-millisecond) activity changes. Thus, they are not able to provide information on the temporal sequence of neural activation that is associated with visceral sensation and pain. The recently introduced MEG technique is helpful in addressing these important issues. The technique is based on recording the magnetic field that is generated by active cortical neurons. Unlike PET and fMRI, MEG is able to record neural activity with milliseconds temporal resolution while identifying the loci of this activation at a level of accuracy comparable to PET and fMRI [9]. A relevant example of the use of MEG is reported in a recently published study [10] using the MEG technique to investigate the brain response to experimental esophageal pain induced by electrical stimuli (fig. 1). In this study, visceral (esophageal) pain was found to be processed in the somatosensory regions as well as in brain regions that are associated with the affective response to pain, i.e. the insula and cingulated cortex. In addition to the localization of the brain response, the investigators were able to show that the affective associated brain areas were activated significantly later than the somatosensory regions. This interesting finding supports a previous finding that a different temporal activation occurs for exogenous (stimulus-dependent) and endogenous (non-stimulus-specific) central pain processing [11].

\section{Functional Brain Imaging in FGID}

Several groups of researchers have used functional brain imaging to investigate the cortical processing of visceral stimulation in healthy individuals and have de- 


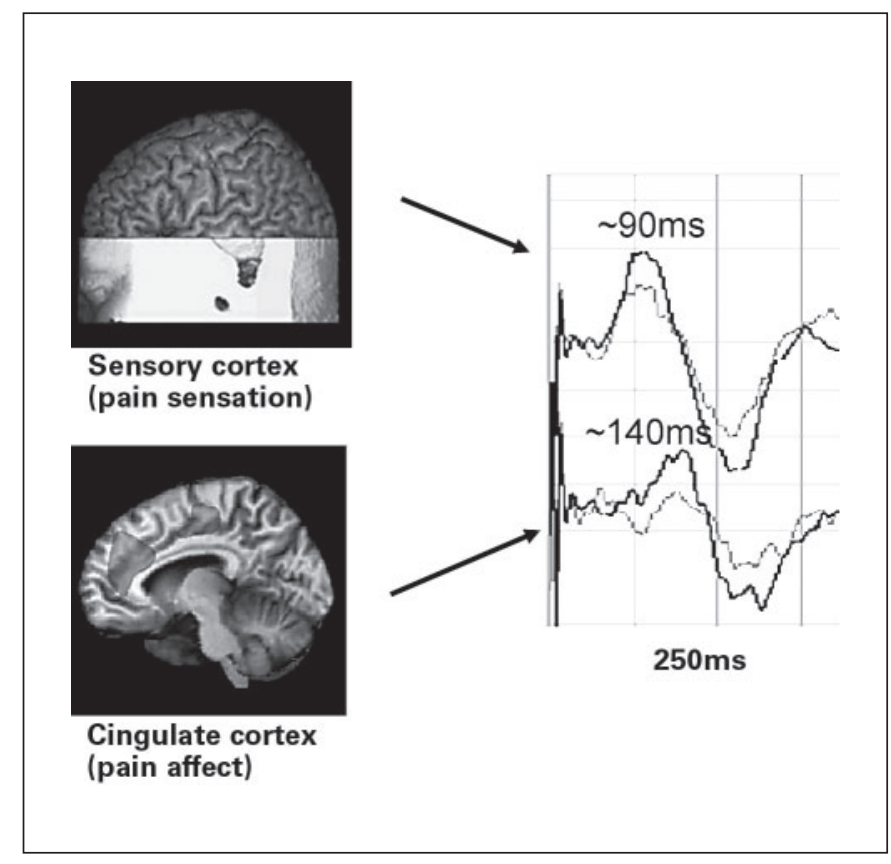

Fig. 1. Sequence of cortical response to visceral stimulation by MEG. Evoked field response recorder with MEG after painful and non-painful electrical stimulation of the esophagus. The top trace is recorded from a virtual electrode positioned in the lateral portion of the primary somatosensory cortex (S1). The lower trace is recorded from a virtual electrode positioned in the perigenual cingulated cortex (pACC). Note that the earlier response in the region associated with sensory discriminatory aspects of pain (S1) is followed by a later response in the region associated with the affective/cognitive processing of pain (pACC) (peak latency responses 90 and $140 \mathrm{~ms}$, respectively). From Hobson et al. [10], with permission.

scribed cortical activation patterns in response to stimulation of various parts of the GI tract, including intraluminal stimulation of the esophagus [10, 12, 13], distal stomach [14-16] and rectum [17-20]. In general, several areas were consistently found to be activated in response to visceral stimulation. These include the insula and the dorsal aspects of the anterior cingulate cortex (dACC). Variable degrees of activation were also found in the primary and secondary somatosensory cortex, the thalamus, and the limbic and paralimbic regions. More detailed information on central representation of visceral afferent signals using functional brain imaging is provided elsewhere [21, 22].

The growing interest in the use of brain imaging research in patients with FGID relates to the evolving concept of visceral hypersensitivity. Multiple studies have demonstrated that patients with irritable bowel syndrome
(IBS) sense noxious and non-noxious intestinal sensation at significantly lower pressures and volumes than healthy controls and have suggested that this abnormally enhanced perception of visceral stimulation is an important pathophysiological mechanism of IBS [23]. The precise mechanisms of visceral hypersensitivity are not yet clear. However, there is growing evidence that central functions, mediated by various psychosocial factors, can affect pain perception even more than peripheral signals. For example, we have recently shown that patients with functional bowel disorders who have a history of abuse report greater pain despite having a lower sensation of rectal balloon distention [24].

The data on the brain response to visceral stimulation in patients with FGID are still limited. Published studies comparing patients with IBS to healthy controls have primarily been descriptive and have focused on identifying differences in regional brain activation in response to non-painful and painful intestinal stimulation. In the first study in this field, Silverman et al. [17] used $\mathrm{H}_{2}{ }^{15} \mathrm{O}$-PET imaging to investigate the brain's response to painful visceral stimulation. In response to rectal distention, patients with IBS showed decreased activation of the perigenual region of the ACC compared with healthy controls. The investigators interpreted this as possible evidence for a dysfunction in the descending inhibitory pathways, which normally down-regulate pain at the level of the spinal cord. They also reported that patients with IBS showed increased activation of the prefrontal cortex (an area associated with increased attention and vigilance response) compared with healthy controls. Several studies using either PET or fMRI have been done since this landmark report and have also documented differences between patients with IBS and healthy controls at increasing levels of accuracy. However, overall the results of the reported studies are not consistent. For example, using a similar protocol, we replicated Silverman's first PET study and found similar findings with respect to the ACC, but we did not identify greater activation of the prefrontal cortex in patients with IBS. Instead, we observed greater activation of the thalamus in response to painful rectal distention [18]. In contrast, Mertz et al. [19] used fMRI and found greater perigenual ACC activation during painful rectal stimulation in patients with IBS but not in healthy controls. As in our study, they found higher thalamic activation in the IBS group and no significant differences in the prefrontal cortex. Results of a PET study by Naliboff et al. [25] showed lower activation of the perigenual ACC but enhanced activation of the rostral/caudal ACC and posterior cingulate cortex - an area considered 
to be related to the interaction between emotions and cognition - in patients with IBS in response to both actual and simulated rectal distention. Taken together, it seems that at this point the differences between patients with IBS and healthy controls are not easily interpretable. The discrepancies in the findings from various reported studies may relate to methodology differences (e.g., PET vs. MRI), different study paradigms, selection of subjects, or difficulties in accurately identifying sub-regions within the brain areas of interest $[6,26]$. It is also possible that the apparent inconsistencies between the studies might relate to the strong variability of the brain responses to visceral pain in patients with IBS [27]; that could reflect the heterogeneous pathophysiology of this disorder. Nevertheless, although the results of brain imaging in IBS are not consistent, they do demonstrate differences in brain activation between patients with IBS and healthy controls. Interestingly, the areas that consistently show greater activation in patients with IBS are concerned with either cognitive processing of sensory input, including attention and response selection (dorsal and midcingulate ACC) or areas that are associated with affective and emotional responses such as the perigenual ACC and medial thalamus. The latter are parts of the medial pain system, also called the motivational-affective pain system, which is believed to be responsible for determining the affective response to visceral sensation. These findings suggest a possible dysfunction of the emotional and behavioral components of the brain pain-experience system in IBS.

Other more recent studies using functional brain imaging investigated and documented differences in brain activation between patients with IBS and other GI conditions such as inflammatory bowel disease (IBD) [28, 29 ] and among patients with or without common comorbidities (e.g., fibromyalgia) [30]. Gender differences in brain activation in IBS were also reported [31]. In addition to the information coming from these mostly descriptive studies, research using functional brain imaging is starting to provide important information on possible pathophysiological mechanisms in FGID. A relevant example is the investigation of the effects of various psychosocial factors, such as a history of abuse [32], on regional brain activation in IBS, and the interactions between psychological factors, brain activation, and clinical presentation [33].

Another advancing area in functional brain imaging research relates to developments and use of new tracers for PET imaging. In a recently published study, PET brain imaging was used to measure serotonin (5-HT) syn- thesis in the brain. The neurotransmitter 5-HT is found both in the brain and intestine, and is involved in braingut interactions that affect both motor and sensory functions. Alterations in 5-HT levels and function are proposed in the pathophysiology of FGID, and 5-HT-modulating agents are commonly used in their treatment. In this study, $\alpha-\left[{ }^{11} \mathrm{C}\right]$ methyl- $L$-tryptophan (AMTrp), a synthetic analog of $L$-tryptophan which is an essential precursor of 5-HT, was used as the imaging tracer. The rate at which AMTrp is taken up by serotonergic neurons [34] is indicative of the regional rates of 5-HT synthesis [35]. Using this method, Nakai et al. [34] have investigated brain 5-HT synthesis in patients with IBS. The investigator reported, for the first time, differences in brain 5-HT synthesis rates between the IBS group and age- and sexmatched controls. Female patients with IBS had higher 5-HT synthesis compared with controls. Interestingly, there were also gender-specific differences in 5-HT regional brain synthesis within the IBS group. These findings suggest an important role of 5-HT synthesis in the pathophysiology of IBS and may help to explain the reported gender differences in the clinical presentation and outcome of IBS [36].

\section{Brain Imaging Research Related to the CNS Effects on Clinical Symptoms and Response to Treatment}

Research in other areas, mainly psychiatry, has shown that functional brain imaging has the potential to improve diagnosis, guide treatment selection for individual patients [37], and predict response to treatment [38, 39]. With regard to FGID though, there have been only a few studies that have used functional brain imaging to investigate the association between brain function and changes in clinical symptoms and the effect of treatment interventions. We have recently described an association between severity of clinical symptoms and psychosocial state and changes in activation of the cingulate cortex in a patient with IBS [40]. In this case study, significant stress and psychological impairments were associated with low visceral pain threshold and activation of the midcingulate cortex, prefrontal cortex, and somatosensory cortex on brain fMRI scans. However, following clinical and psychological improvements, we observed resolution in the activation in these areas, and this was accompanied by an increase in pain threshold to rectal distention. This outcome suggests that brain activation in areas concerned with visceral pain is related to the 
patient's clinical and psychological status and that this cortical activation may be responsive to clinical improvement.

\section{Placebo Effect}

It is well known that the placebo effect in FGID is considerable [41, 42]. Current data have shown that the brain response to placebo can be similar to its response to an active agent [43]. Therefore, it is important to document and understand the effects of placebo on brain activation in order to correctly interpret the effects of various treatment interventions. With regard to patients with FGID, a recent study by Lieberman et al. [44] identified the central neural outcome of placebo effects. Using PET imaging, Lieberman et al. investigated the brain response to intestinal discomfort before and after a 3-week placebo regimen. They found that an increase in the activation of the right ventrolateral prefrontal cortex predicted selfreported symptom improvement. Furthermore, they were able to show that this relationship was mediated by changes in the dACC region, which is typically associated with the unpleasantness component of pain experience. This supports the authors' theory that the activation of prefrontal regions associated with thinking about negative affect can diminish the reactivity of regions involved in pain unpleasantness, such as dACC and amygdala.

\section{Alosetron}

The use of serotonergic agents in patients with FGID has gained an increasing interest in the last few years. The rationale for their use is based on the critical role that 5HT plays in normal gut function and brain-gut communication. However, only a few studies have examined their central effects. A group of researchers at UCLA has used $\mathrm{H}_{2}{ }^{15} \mathrm{O}$-PET imaging to investigate the effects of alosetron, a $5-\mathrm{HT}_{3}$ receptor antagonist, in IBS. Alosetron treatment (1-4 mg twice daily for 3 weeks) was associated with reduced regional cerebral blood flow in several $5-\mathrm{HT}_{3}$ receptor-containing regions of the brain, including frontotemporal and various limbic structures (including amygdala, ventral striatum, and dorsal pons). Furthermore, in this study, the drug-induced reduction in symptoms correlated with a decrease in the activity of central networks mediating emotional experience to pain [45, 46]. Another recently published study investigated the effects of alosetron on brain 5-HT synthesis in patients with non-constipation IBS. 5-HT synthesis (assessed by PET with AMTrp as tracer) was greater in several brain regions after alosetron treatment than after the placebo treatment. However, unlike males who showed greater synthesis in the parahippocampal gyrus and cerebellum following alosetron treatment, the female subjects had greater 5-HT synthesis in the posterior cingulate gyrus [47]. These interesting gender-treatment interactions of brain 5-HT synthesis may be related to the previously observed gender differences in the clinical response to alosetron.

\section{Antidepressants}

Tricyclic antidepressants (TCAs) are commonly used in the treatment of chronic, painful conditions including IBS and have been shown to have a greater therapeutic effect than placebo [48]. However, the mechanisms associated with their beneficial effects in these patients are unclear. The TCA reduction in intestinal pain sensitivity may relate to central psychotropic effects via their action on CNS norepinephrine and 5-HT transporters and/or to peripheral effects on GI physiology. A recently published functional brain imaging study has started to shed some light on this issue. Morgan et al. [49] have used fMRI to investigate the effects of the TCA amitriptyline on ACC activation in patients with IBS. Female patients were randomized to amitriptyline $50 \mathrm{mg} /$ day or placebo for 1 month in a crossover alternate treatment trial. Cerebral activation during rectal distention was traced using fMRI, and a comparison was made between the placebo and amitriptyline groups. Amitriptyline was found to reduce pain-related activation in the perigenual (limbic) ACC and left posterior parietal cortex. However, the reductions in brain activation in these affective and cognitive regions were only seen during stress and not during relaxation. The authors suggested that these findings indicate that amitriptyline works in the CNS rather than peripherally and that it blunts pain and other symptoms associated with psychological stress.

\section{Cognitive-Behavioral Therapy}

Over the last 15 years, there have been a series of trials evaluating various combinations of behavioral and cognitive procedures for treatment of IBS, and several studies provided evidence for their beneficial effects in IBS [48, 50]. The effect of cognitive therapy on regional brain activation in patients with IBS was recently investigated by Lackner et al. [51] at the University at Buffalo (fig. 2). The investigators used $\mathrm{H}_{2}{ }^{15} \mathrm{O}-\mathrm{PET}$ imaging to identify brain regions that underlie symptom changes with cognitive therapy. Post-treatment scans showed reduced activity in corticolimbic regions that subserve hypervigilance and emotion regulation, including the parahippocampal 


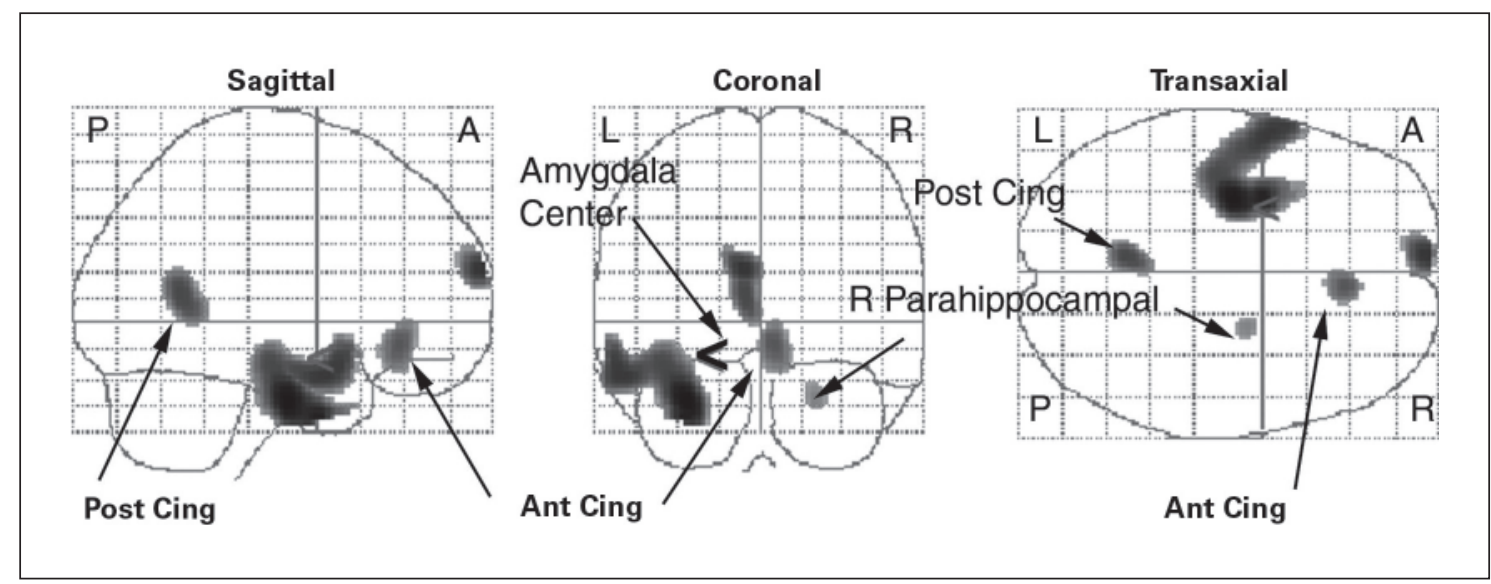

Fig. 2. PET imaging showing reduction in neural activity after cognitive therapy. Projections of $\mathrm{p}$ values for SPM contrast (pre-therapy) - (post-therapy) are shown with a threshold $p=0.001(p<0.005)$, uncorrected for multiple comparisons. The $<$ symbol, shown best on the coronal projection, marks the center of the a priori defined sphere delineating the left amygdala. Ant Cing = Anterior cingulate; Post Cing = posterior cingulate; $\mathrm{R}=$ right; $\mathrm{L}=1 \mathrm{eft}$; $\mathrm{A}=$ anterior; $\mathrm{P}=$ posterior. From Lackner et al. [51], with permission.

gyrus and inferior portion of the right cingulate cortex. Most important, these limbic activity changes were accompanied by parallel improvements in patients' symptoms, including pain, bowel dysfunction, and psychological variables such as anxiety and worry. These findings suggest a possible physiological mechanism by which cognitive therapy induces symptom improvement in IBS.

\section{Conclusions}

Functional brain imaging has greatly enhanced the ability to investigate brain-gut interactions and to assess the CNS role on pain perception. The emerging capability of neuroimaging techniques for exploring a broad range of thoughts, mood, and emotions makes it an extremely useful research tool for studying FGID. However, the multifactorial, multideterminant nature of the disorder, current limitations on the understanding of FGID pathophysiology, and the heterogeneous patient population make research in this field complex and difficult. Despite these limitations, studies using functional brain imaging were able to demonstrate differences in regional brain activation between patients with IBS and control subjects as well as between subgroup of patients with IBS. The more recent studies are starting to shed light on pathophysiological mechanisms that underlie the generation of functional GI symptoms as well as the response to treatment. The continued development of brain imaging techniques provides not only exciting opportunities but also significant challenges to the field. The critical next step is to move from descriptive-only to hypothesis-driven and mechanistic studies. This will require the development of better multivariate statistical strategies that will enable researchers to optimally integrate the multiple physiological and psychosocial factors that influence both brain activation and clinical presentation into the analysis of brain imaging studies in FGID. Future research in this area is likely to provide valuable insight into the pathophysiology of FGID and to increase understanding of the neurophysiological mechanisms that are involved in the generation of these disorders. This will lead to improving clinical diagnosis, identification of individual candidates for selected treatments, and development of better and more effective pharmacological treatments. 


\section{References}

1 Ringel Y, Drossman DA: Irritable bowel syndrome: classification and conceptualization. J Clin Gastroenterol 2002;35:S7-S10.

-2 Ringel Y, Sperber AD, Drossman AD: Irritable bowel syndrome. Annu Rev Med 2001;52: 319-338.

-3 Whitehead WE, Palsson O, Jones KR: Systematic review of the comorbidity of irritable bowel syndrome with other disorders: what are the causes and implications? Gastroenterology 2002;122:1140-1156.

4 Palsson OS, Drossman DA: Psychiatric and psychological dysfunction in irritable bowel syndrome and the role of psychological treatments. Gastroenterol Clin North Am 2005;34: 281-303.

5 Van Oudenhove L, Demyttenaere K, Tack J, Aziz Q: Central nervous system involvement in functional gastrointestinal disorders. Best Pract Res Clin Gastroenterol 2004; 18:663-680.

6 Ringel Y: Brain research in functional gastrointestinal disorders. J Clin Gastroenterol 2002; 35(suppl):S23-S25.

7 Villringer A: Understanding functional neuroimaging methods based on neurovascular coupling. Adv Exp Med Biol 1997;413:19771993.

-8 Lin W, An H, Chen Y, Nicholas P, Zhai G, Gerig G, Gilmore J, Bullitt E: Practical consideration for $3 \mathrm{~T}$ imaging. Magn Reson Imaging Clin N Am 2003;11:615-639.

-9 Vrba J, Robinson SE: Signal processing in magnetoencephalography. Methods 2001;25:249271.

$\checkmark 10$ Hobson AR, Furlong PL, Worthen SF, Hillebrand A, Barnes GR, Singh KD, Aziz Q: Realtime imaging of human cortical activity evoked by painful esophageal stimulation. Gastroenterology 2005;128:610-619.

11 Bromm B, Lorenz J: Neurophysiological evaluation of pain. Electroencephalogr Clin Neurophysiol 1998;107:227-253

-12 Kern MK, Birn RM, Jaradeh S, Jesmanowicz A, Cox RW, Hyde JS, Shaker R: Identification and characterization of cerebral cortical response to esophageal mucosal acid exposure and distention. Gastroenterology 1998;115: 1353-1362.

$\checkmark 13$ Aziz Q, Andersson R, Valind S, Sundin A, Hamdy S, Jones AKP, Foster E, Langstrom B, Thompson DG: Identification of human brain loci processing esophageal sensation using positron emission tomography. Gastroenterology 1997;113:50-59.

14 Ladabaum U, Minoshima S, Hasler WL, Cross D, Chey WD, Owyang C: Gastric distension correlates with activation of multiple cortical and subcortical regions. Gastroenterology 2001;120:369-376.

15 Vandenbergh J, Dupont P, Fischler B, Bormans G, Persoons P, Janssens J, Tack J: Regional brain activation during proximal stomach distention in humans: a positron emission tomography study. Gastroenterology 2005; 128:564-573.
16 Lu CL, Wu YT, Yeh TC, Chen LF, Chang FY, Lee SD, Ho LT, Hsieh JC: Neuronal correlates of gastric pain induced by fundus distension: a 3T-fMRI study. Neurogastroenterol Motil 2004; 16:575-587.

-17 Silverman DH, Munakata JA, Ennes H, Mandelkern MA, Hoh CK, Mayer EA: Regional cerebral activity in normal and pathological perception of visceral pain. Gastroenterology 1997;112:64-72.

18 Ringel $\mathrm{Y}$, Drossman AD, Turkington TG, Hawk TC, Bradshaw B, Coleman RE, Whitehead WE: Dysfunction of the motivational-affective pain system in patients with IBS: PET imaging in response to rectal balloon distension. Gastroenterology 2000B; 118:A444.

19 Mertz H, Morgan V, Tanner G, Pickens D, Price R, Shyr Y, Kessler R: Regional cerebral activation in irritable bowel syndrome in control subjects with painful and nonpainful rectal distention. Gastroenterology 2000;118:842848.

20 Kern MK, Arndorfer RC, Hyde JS, Shaker R: Cerebral cortical representation of external anal sphincter contraction: effect of effort. Am J Physiol 2004;286:G304-G311.

21 Derbyshire SW: Visceral afferent pathways and functional brain imaging. ScientificWorldJournal 2003;3:1065-1080.

22 Bonaz B: Visceral sensitivity perturbation integration in the brain-gut axis in functional digestive disorders. J Physiol Pharmacol 2003; 54(suppl 4):27-42.

-23 Mertz H, Naliboff B, Munakata J, Niazi N, Mayer EA: Altered rectal perception is a biological marker of patients with irritable bowel syndrome. Gastroenterology 1995;109:40-52.

-24 Ringel Y, Whitehead WE, Toner BB, Diamant NE, Hu Y, Jia H, Bangdiwala SI, Drossman DA: Sexual and physical abuse are not associated with rectal hypersensitivity in patients with irritable bowel syndrome. Gut 2004;53: 838-842.

25 Naliboff BD, Derbyshire SWG, Munakata J, Berman S, Mandelkem MA, Chang L, Mayer EA: Cerebral activation in irritable bowel syndrome patients and control subjects during rectosigmoid stimulation. Psychosom Med 2001; 63:365-375.

26 Mayer EA, Derdyshire S, Naliboff BD: Cerebral activation in irritable bowel syndrome. Gastroenterology 2000;119:1418.

27 Bonaz B, Baciu M, Papillon E, Bost R, Gueddah N, Le Bas JF, Fournet J, Segebarth C: Central processing of rectal pain in patients with irritable bowel syndrome: an fMRI study. Am J Gastroenterol 2002;97:654-661.

28 Bernstein CN, Frankenstein UN, Rawsthorne P, Pitz M, Summers R, McIntyre MC: Cortical mapping of visceral pain in patients with GI disorders using functional magnetic resonance imaging. Am J Gastroenterol 2002;97:319327.
29 Mayer EA, Berman S, Suyenobu B, Labus J, Mandelkern MA, Naliboff BD, Chang L: Differences in brain responses to visceral pain between patients with irritable bowel syndrome and ulcerative colitis. Pain 2005;115:398409.

30 Chang L, Berman S, Mayer EA, Suyenobu B, Derbyshire S, Naliboff B, Vogt B, FitzGerald L, Mandelkern MA: Brain responses to visceral and somatic stimuli in patients with irritable bowel syndrome with and without fibromyalgia. Am J Gastroenterol 2003;98:1354-1361.

- 31 Naliboff BD, Berman S, Chang L, Derbyshire SW, Suyenobu B, Vogt BA, Mandelkern M, Mayer EA: Sex-related differences in IBS patients: central processing of visceral stimuli. Gastroenterology 2003;124:1738-1747.

32 Ringel Y, Drossman DA, Turkington TG, Bradshaw B, Hawk TC, Bangdiwala S, Coleman RE, Whitehead WE: Regional brain activation in response to rectal distension in patients with irritable bowel syndrome and the effect of a history of abuse. Dig Dis Sci 2003; 48:1774-1781.

33 Ringel Y, Drossman DA, Leserman J, Lin W, Liu H, Vogt B, Whitehead WE: Association of anterior cingulate cortex activation with psychosocial distress and pain reports. Gastroenterology 2003;124:A97.

- 34 Nakai A, Kumakura Y, Boivin M, Rosa P, Diksic M, D’Souza D, Kersey K: Sex differences of brain serotonin synthesis in patients with irritable bowel syndrome using $\alpha-\left[{ }^{11} \mathrm{C}\right]$ methyl$L$-tryptophan, positron emission tomography and statistical parametric mapping. Can J Gastroenterol 2003;17:191-196.

35 Diksic M: Labelled $\alpha$-methyl- $L$-tryptophan as a tracer for the study of the brain serotonergic system. J Psychiatry Neurosci 2001;26:293303.

36 Chang L, Heitkemper MM: Gender differences in irritable bowel syndrome. Gastroenterology 2002;123:1686-1701.

37 Mayberg HS: Modulating dysfunctional limbic-cortical circuits in depression: towards development of brain-based algorithms for diagnosis and optimised treatment. Br Med Bull 2003;65:193-207.

- 38 Mayberg HS, Brannan SK, Mahurin RK, Jerabek PA, Brickman JS, Tekell JL, Silva JA, McGinnis S, Glass TG, Martin CC, Fox PT: Cingulate function in depression: a potential predictor of treatment response. Neuroreport 1997;8:1057-1061.

39 Ketter TA, Wang PW: Predictors of treatment response in bipolar disorders: evidence from clinical and brain imaging studies. J Clin Psychiatry 2002;63(suppl 3):21-25.

40 Drossman DA, Ringel Y, Vogt BA, Leserman J, Lin W, Smith JK, Whitehead W: Alterations of brain activity associated with resolution of emotional distress and pain in a case of severe irritable bowel syndrome. Gastroenterology 2003;124:754-761. 
41 Spiller RC: Problems and challenges in the design of irritable bowel syndrome clinical trials: experience from published trials. Am J Med 1999; 107:91S-97S.

42 Talley NJ: Evaluation of drug treatment in irritable bowel syndrome. Br J Clin Pharmacol 2003;56:362-369.

43 Mayberg HS, Silva JA, Brannan SK, Tekell JL, Mahurin RK, McGinnis S, et al: The functional neuroanatomy of the placebo effect. Am J Psychiatry 2002;159:728-737.

44 Lieberman MD, Jarcho JM, Berman S, Naliboff BD, Suyenobu BY, Mandelkern M, Mayer EA: The neural correlates of placebo effects: a disruption account. Neuroimage 2004;22: 447-455.

45 Berman SM, Chang L, Suyenobu B, Derbyshire SW, Stains J, Fitzgerald L, Mandelkern M Hamm L, Vogt B, Naliboff BD, Mayer EA: Condition-specific deactivation of brain regions by $5-\mathrm{HT}_{3}$ receptor antagonist alosetron. Gastroenterology 2002;123:969-977.
46 Mayer EA, Berman S, Derbyshire SW, Suyenobu B, Chang L, Fitzgerald L, Mandelkern M, Hamm L, Vogt B, Naliboff BD: The effect of the $5-\mathrm{HT}_{3}$ receptor antagonist, alosetron, on brain responses to visceral stimulation in irritable bowel syndrome patients. Aliment Pharmacol Ther 2002; 16:1357-1366.

47 Nakai A, Diksic M, Kumakura Y, D’Souza D, Kersey K: The effects of the 5- $\mathrm{HT}_{3}$ antagonist, alosetron, on brain serotonin synthesis in patients with irritable bowel syndrome. Neurogastroenterol Motil 2005; 17:212-221.

48 Drossman DA, Toner BB, Whitehead WE, Diamant NE, Dalton CB, Duncan S, Emmott S, Proffitt V, Akman D, Frusciante K, Le T, Meyer K, Bradshaw B, Mikula K, Morris CB Blackman CJ, Hu Y, Jia H, Li JZ, Koch GG, Bangdiwala SI: Cognitive-behavioral therapy versus education and desipramine versus placebo for moderate to severe functional bowel disorders. Gastroenterology 2003;125: 19-31.
49 Morgan V, Pickens D, Gautam S, Kessler R, Mertz H: Amitriptyline reduces rectal pain-related activation of the anterior cingulate cortex in patients with irritable bowel syndrome. Gut 2005;54:601-607.

50 Kennedy T, Jones R, Darnley S, Seed P, Wessely S, Chalder T: Cognitive behaviour therapy in addition to antispasmodic treatment for irritable bowel syndrome in primary care: randomised controlled trial. BMJ 2005;331: 435

$>51$ Lackner JM, Lou Coad M, Mertz HR, Wack DS, Katz LA, Krasner SS, Firth R, Mahl TC, Lockwood AH: Cognitive therapy for irritable bowel syndrome is associated with reduced limbic activity, GI symptoms, and anxiety. Behav Res Ther 2006;44:621-638. 